\title{
Editorial: Coordination of mRNA Transport and Translation With Vesicle and Organelle Trafficking and Dynamics
}

\author{
Anni Vedeler* and Clive R. Bramham* \\ Department of Biomedicine, University of Bergen, Bergen, Norway \\ Keywords: RNP granule, extracellular vesicles, mRNA, endosomes, lysosomes
}

\section{Editorial on the Research Topic}

Coordination of mRNA Transport and Translation With Vesicle and Organelle Trafficking and Dynamics

\section{OPEN ACCESS}

Edited by:

Vladimir Lupashin,

University of Arkansas for Medical

Sciences, United States

Reviewed by:

Kentaro Hanada,

National Institute of Infectious

Diseases (NIID), Japan

*Correspondence:

Anni Vedeler

Anni.Vedeler@biomed.uib.no

Clive R. Bramham

Clive.Bramham@biomed.uib.no

Specialty section:

This article was submitted to

Membrane Traffic,

a section of the journal

Frontiers in Cell and Developmental

Biology

Received: 22 October 2021

Accepted: 29 October 2021

Published: 18 November 2021

Citation:

Vedeler A and Bramham CR (2021)

Editorial: Coordination of mRNA

Transport and Translation With Vesicle

and Organelle Trafficking

and Dynamics.

Front. Cell Dev. Biol. 9:800136.

doi: $10.3389 /$ fcell.2021.800136
Mammalian cells have complex endomembrane systems and vesicular and tubular transport routes that connect the cellular internal compartments and establish the biosynthetic and endocytic pathways. Originally, the sorting and transport activities of these pathways, by ensuring the asymmetric distribution of endoplasmic reticulum (ER)-derived proteins and lipids, were thought to provide the cells with the ability to build distinct functional domains. However, studies carried out during the last decades have revealed that functional polarity of cells can be determined already at the post-transcriptional level via the sorting and transport of mRNAs (Martin and Ephrussi, 2009). Thus, specific sets of mRNAs together with RNA-binding proteins in dynamic ribonucleoprotein complexes, called RNP granules, can move in a motor-dependent manner along cytoskeletal tracks from the cell center to peripheral locations docked onto moving vesicles (Kharod et al.). Classical examples of these processes include long-range microtubule-based transport of selected mRNAs via membraneless RNP granules, allowing fast synaptic re-modeling (Bramham and Wells, 2007; Holt et al., 2019; Dalla Costa et al., 2021). As summarized in this Research Topic volume, recent work now reveals coordinate delivery of RNP granules with membranous organelles such as endosomes and lysosomes to neuronal axons and dendrites, as well as transport of mitochondrial mRNAs inside endosomes (Kharod et al.; Rajgor et al.). The formation of RNP granules occurs via liquid-liquid phase separation (Rajgor et al.). Namely, RNP granules lack a membrane and thus phase separation occurs via highly dynamic RNA - protein interactions. The coiled-coil structure is significantly overrepresented in RNA-binding proteins, as found by prediction using databases combined with different algorithms (softwares), and often localized to liquid-liquid phase separated neuronal granules (Ford and Fioriti). The quest to visualize the regulation and function of RNP granules in situ has been enormously facilitated by developments in optics and genetically-encoded probes, allowing detection of nascent mRNA and protein at the single molecule level (Kharod et al.; Ford and Fioriti). The RNA-binding motifs and the coiled-coil structure found in RNA-binding proteins are in proximity but never overlapping (Ford and Fioriti) suggesting that the coil-coiled motifs are involved in interaction with protein ligands. Interestingly, it has been proposed that coiled-coil oligomerization under certain circumstances may lead to $\beta$-sheet formation, which in turn may lead to the formation of insoluble aggregates and various neurodegenerative diseases (Ford and Fioriti).

Interestingly, it was shown that a subset of RNP granules containing mRNAs necessary for growth cone morphology, tether onto lysosomes via Annexin A11, and the two organelles are transported 
together in cortical neurons. Amyotrophic lateral sclerosis (ALS)associated mutations in Annexin A11 disrupted this coordinated transport and contribute to the development of ALS (Liao et al., 2019) (Kharod et al.; Rajgor et al.; Ford and Fioriti). The annexin family may turn out to be an interesting protein family in the coordination and regulation of vesicle and mRNA transport/ translation as at least one member of the family of $\mathrm{Ca}^{2+}$ dependent lipid-binding proteins, Annexin A2, is involved in the localization (Mickleburgh et al., 2005) of c-myc mRNA and binds to the internal ribosomal entry sites (IRESs) of c-myc (Strand et al., 2021) and p53 (Sharathchandra et al., 2012) mRNAs.

Once transported to the synapse, translation of the mRNAs occurs by an activity-dependent mechanism and is fine-tuned by miRNAs and fueled by nearby mitochondria (Kharod et al.; Rajgor et al.). Post-translational modifications of the proteins in the RNP granules play a key role in releasing the mRNAs from the granules to be actively translated at the synapse (Rajgor et al.). Thus, local protein synthesis allows the cells to rapidly respond to external signals on-site.

The co-ordination of mRNA transport with endosomal/ lysosomal transport is important for intracellular communication while transport of lipids, proteins, and RNA in extracellular vesicles (EVs) is important for intercellular communication (Prieto-Vila et al.). EVs consist of microvesicles released by budding from the plasma membrane, apoptotic bodies, and exosomes derived from the endocytic pathway by release of intraluminal vesicles from multivesicular bodies at the plasma membrane. Diseases, including cancer, appear to increase the amount of released/shed EVs. The mRNA cargo and other types of RNA in EVs can modulate physiological responses in the recipient cells. It has been shown that the mRNAs are translated, and the protein product exerts functional effects in recipient cells (Prieto-Vila et al.). Specific elements in both mRNA and miRNA control the loading of these RNAs into EVs, thus specifically targeting them for intercellular communication. In addition to the ESCRT protein machinery, specific proteins such as activity-regulated cytoskeleton associated protein (Arc) in neurons and LC3/

\section{REFERENCES}

Ashley, J., Cordy, B., Lucia, D., Fradkin, L. G., Budnik, V., and Thomson, T. (2018). Retrovirus-Like Gag Protein Arcl Binds RNA and Traffics across Synaptic Boutons. Cell 172 (1-2), 262-274. e211. doi:10.1016/j.cell.2017.12.022

Bramham, C. R., and Wells, D. G. (2007). Dendritic mRNA: Transport, Translation and Function. Nat. Rev. Neurosci. 8 (10), 776-789. doi: $10.1038 / \mathrm{nrn} 2150$

Dalla Costa, I., Buchanan, C. N., Zdradzinski, M. D., Sahoo, P. K., Smith, T. P., Thames, E., et al. (2021). The Functional Organization of Axonal mRNA Transport and Translation. Nat. Rev. Neurosci. 22 (2), 77-91. doi:10.1038/ s41583-020-00407-7

Eriksen, M. S., Nikolaienko, O., Hallin, E. I., Grødem, S., Bustad, H. J., Flydal, M. I., et al. (2021). Arc Self-Association and Formation of Virus-Like Capsids Are Mediated by an N-Terminal Helical Coil Motif. Febs J. 288 (9), 2930-2955. doi: $10.1111 /$ febs. 15618
ATG8 in the autophagy pathways, participate in loading of RNA into EVs. Arc self-assembles into virus-like capsids which harbor Arc mRNA inside (Ashley et al., 2018; Pastuzyn et al., 2018). The assembly of capsids is also promoted by Arc protein interaction with RNA, mediated by a coiled-coil motif (Eriksen et al., 2021). Tumor-derived EVs appear to play a role in all stages of cell transformation, such as metastasis, neo-angiogenesis, and drug resistance. Furthermore, they prime the tumor microenvironment to become more favorable for the tumor (Prieto-Vila et al.). EVs also appear to be involved in immunosuppression. Specific mRNAs from tumor-derived EVs can be used as biomarkers for cancer diagnosis and for treatment prognosis. Thus, inhibition of EVs has been considered as a therapeutic approach but this is still a method to be developed further (Prieto-Vila et al.).

Thus, it has become evident that membrane traffic and mRNA transport in mammalian cells are coordinated processes, which not only share common pathways and regulatory factors, but also respond to the same extracellular signals. These closely coordinated processes ensure specificity in terms of the mRNAs involved and are highly energy efficient, which becomes particularly evident in the gigantic neurons. Also, the communication of cells with their environment is tightly regulated and can be modulated by the protein and RNA cargoes enclosed in the EVs. As pointed out by the papers of this Frontiers Research Topic, several outstanding questions remain to be addressed by future work. Thus, the era of the present topic: "Coordination of mRNA transport and translation with vesicle and organelle trafficking and dynamics" is only at its dawn and much effort is needed to reveal the post-translational modifications involved the joint regulation of these processes, as well as to identify the specific proteins acting at the crossroads of the coordinated transport events.

\section{AUTHOR CONTRIBUTIONS}

All authors listed have made a substantial, direct, and intellectual contribution to the work and approved it for publication.

Holt, C. E., Martin, K. C., and Schuman, E. M. (2019). Local Translation in Neurons: Visualization and Function. Nat. Struct. Mol. Biol. 26 (7), 557-566. doi:10.1038/s41594-019-0263-5

Liao, Y.-C., Fernandopulle, M. S., Wang, G., Choi, H., Hao, L., Drerup, C. M., et al. (2019). RNA Granules Hitchhike on Lysosomes for Long-Distance Transport, Using Annexin A11 as a Molecular Tether. Cell 179 (1), 147-164. e120. doi:10.1016/j.cell.2019.08.050

Martin, K. C., and Ephrussi, A. (2009). mRNA Localization: Gene Expression in the Spatial Dimension. Cell 136 (4), 719-730. doi:10.1016/j.cell.2009.01.044

Mickleburgh, I., Burtle, B., Hollås, H., Campbell, G., Chrzanowska-Lightowlers, Z., Vedeler, A., et al. (2005). Annexin A2 Binds to the Localization Signal in the $3^{\prime}$ Untranslated Region of C-Myc mRNA. Febs j 272 (2), 413-421. doi:10.1111/ j.1742-4658.2004.04481.x

Pastuzyn, E. D., Day, C. E., Kearns, R. B., Kyrke-Smith, M., Taibi, A. V., McCormick, J., et al. (2018). The Neuronal Gene Arc Encodes a Repurposed Retrotransposon Gag Protein that Mediates Intercellular 
RNA Transfer. Cell 172 (1-2), 275-288, e218, doi:10.1016/ j.cell.2017.12.024

Sharathchandra, A., Lal, R., Khan, D., and Das, S. (2012). Annexin A2 and PSF Proteins Interact with P53 IRES and Regulate Translation of P53 mRNA. RNA Biol. 9 (12), 1429-1439. doi:10.4161/rna.22707

Strand, E., Hollås, H., Sakya, S. A., Romanyuk, S., Saraste, M. E. V., Grindheim, A. K., et al. (2021). Annexin A2 Binds the Internal Ribosomal Entry Site of C-Myc mRNA and Regulates its Translation. RNA Biol., 1-18. doi:10.1080/15476286.2021.1947648

Conflict of Interest: The authors declare that the research was conducted in the absence of any commercial or financial relationships that could be construed as a potential conflict of interest.
Publisher's Note: All claims expressed in this article are solely those of the authors and do not necessarily represent those of their affiliated organizations, or those of the publisher, the editors and the reviewers. Any product that may be evaluated in this article, or claim that may be made by its manufacturer, is not guaranteed or endorsed by the publisher.

Copyright (C) 2021 Vedeler and Bramham. This is an open-access article distributed under the terms of the Creative Commons Attribution License (CC BY). The use, distribution or reproduction in other forums is permitted, provided the original author(s) and the copyright owner(s) are credited and that the original publication in this journal is cited, in accordance with accepted academic practice. No use, distribution or reproduction is permitted which does not comply with these terms. 